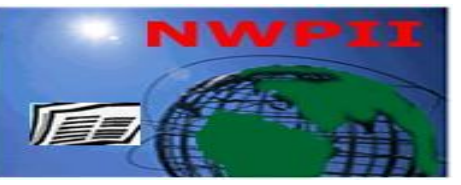

American Journal of Biomedical Sciences

ISSN: 1937-9080

nwpii.com/ajbms

\title{
Slippage Sensory Feedback and Nonlinear Force Control System for a Low- Cost Prosthetic Hand
}

\author{
C.F. Pasluosta, H. Tims and A.W.L. Chiu*
}

College of Engineering and Science, Biomedical Engineering Department, Louisiana Tech University, Ruston, LA, USA.

*Corresponding Author

Dr. Alan Chiu

College of Engineering and Science

Biomedical Engineering Department

Louisiana Tech University

Ruston, LA, USA.

Tel: 318-257-5231

E-mail: alanchiu@latech.edu

Received: 17 March 2009; | Revised: 19 May 2009; | Accepted: 3 June 2009

\begin{abstract}
The low acceptability of current prosthetic devices can be attributed to the extensive psychological effort and the high cost associated with them. To address these concerns, an on-line slippage detector was developed using only inexpensive force sensors placed at the tips of a prototype hand. The prototype consists of a five fingered prosthetic hand consisting of active digits driven independently by DC motors. Force sensor resistors (FSR) are placed at the tip of each active finger and potentiometers are attached at the proximal and middle joints. Using the information from the FSR, not only can we detect the level of normal force exerted but also slippage between the fingers and the object by calculating the fluctuations of the exerted force. An on-line algorithm is developed to calculate the derivative of the force and determine when slippage is produced. Nonlinear model predictive control (NMPC) is used to provide feedback control to the prosthetic device. It utilizes a neural network to model the dynamics of each finger. Using this model, it is possible to predict future plant performance (the amount of force exerted by the prosthetic hand). Consequently, the controller uses this prediction to calculate the best input (current needed to drive the actuators) for the system to obtain the desired output over a specific time horizon. In order to calculate the future control inputs, the optimization system minimizes the cost function associated with the difference between the measured force and the reference / target output. Experimental protocols involve grasping various objects and inducing slippage. Data was collected using the NI DAQ cards and LabVIEW software. Experiments showed promising results using this strategy in which the force exerted on an object can be modulated without additional efforts from the users.
\end{abstract}

Keywords: Prosthetic hand; Slippage; Neural networks; Nonlinear Control. 


\section{Introduction}

The lost of a limb produces a tremendous psychological, social and economical impact for the affected individual. Over the past decades, new upperlimb prosthetic devices have been developed to increase the life quality of people with limb disabilities. Moreover, the development of EMGcontrolled prosthetic hands has increased the dexterousness of current prostheses by achieving more degrees of freedom (DoF) in motions and functions. However, about $35 \%$ of the affected people do not use their prosthetic devices regularly [1]. One major limitation to translating these advanced technologies to the end users is the high cost associated with these devices. Beside the economical aspect, an EMGcontrolled prosthetic hand must satisfy some design conditions. First, the device must be simple to use in that it must be easily adaptable for different users with reduced psychological effort and muscle fatigue. Second, the device must be able to control enough DoF to allow various types of grasping configurations at various strengths. Finally, electromechanical considerations (such as weight, dimensions and power consumption) must be taken into account for practical purposes [2].

With the aim of accomplishing the abovementioned requirements, we propose an automatic feedback control strategy for an EMGcontrolled prosthetic device. The feedback control signals are obtained by using inexpensive sensors placed in strategically selected locations on the prosthetic device. This information is monitored continuously and utilized to drive low-cost actuators in order to safely and securely hold different objects. Reduction in hardware complexity and increased sophistication of the software is designed to lower the overall cost.

The proposed control strategy is divided in four logic states: Contact, Hold, Detection and Release. This kind of grasping methodology has been successfully reported in the literature [3, 4]. At the Contact state the hand is closed until a force threshold is reached. The system is then switched to the Hold state where stable grasping configuration is produced. The system then alternates between the Hold state and the Detection state to continuously monitor any sliding or unintended movement of the object in order to maintain a secure hold of the object. If slippage is detected, the grasp force is increased until no sliding movement is produced. Subsequently, when the user decides to open the hand, the system switches to the
Release state in which the hand returns to its initial open palm configuration.

The system uses inexpensive force sensor resistor (FSR) to measure the normal force and estimate the onset of slippage. Its utility as a slip detector had been reported by calculating the derivative of the normal force [5]. However, this methodology has not been found yet in prosthetic application.

To ensure the maintenance of a stable grasp in the Hold state, nonlinear model predictive control (NMPC) based on neural networks is implemented. This control technique is selected based on its ability to handle highly nonlinear systems. It is well documented that the control of prosthesis faces certain challenges in nonlinearities caused by the existence of motor dead bands, friction and large gear ratios [6]. Moreover, the utilization of low-cost sensors and actuators, with their consequently low-quality response, introduces additional nonlinearities to the system. To overcome these constraints, the appropriate application of nonlinear control techniques is essential.

In this paper, a low-cost prototype of the prosthetic device is presented along with a novel slip detection technique and a control system to modulate the force exerted by each finger. The experimental validations of controlling the induced slippage as well as the preliminary results of the feedback force control system implementation are also presented.

\section{Materials and methods}

\subsection{Prototype Hand}

A low-cost five fingered underactuated hand with $10 \mathrm{DoF}$ was constructed (Figure 1). It is considered an underactuated device because the number of actuators needed is less than the possible DoF. In this prototype, each finger consists of $2 \mathrm{DoF}$. Each DoF refers to a separate movement and it is not controlled independently. As a result of the underactuated nature of the system, a grasping configuration adaptable to the shape of the object is obtained [2]. It was designed using the SolidWorks CAD software and printed with ABS plastic on a rapid prototyping machine. The thumb, index and middle fingers are the active fingers that are driven independently by three low-cost DC gear motors (MS-16024-050 from BaneBots, Loveland, CO). The ring and little fingers are passive and mechanically connected to the middle finger by mean of gears and these three fingers move all together. Furthermore, the flexion of each finger is controlled by a flexor tendon-cable connected to a pulley. The pulley is attached to a worm-gear system 
driven by the DC motors. In addition, the extension of the fingers is achieved by springs connected to an extensor tendon-cable. All the actuators and gear systems are housing at the palm of the hand. The fingers and the palm are covered with soft foam to increase the grip of the hand and to assist in the slip detection state. The sensory system consisting of inexpensive FSR (Interlink Electronics, Camarillo, $\mathrm{CA}$ ), are placed at the tip of each active finger. Potentiometers are attached at the proximal and middle joints on the active fingers. Finally, motor drivers and signal conditioning circuit complete the mechatronic design of the prototype. The motor driver consists on a pulse width modulator (PWM) implemented by hardware and connected to two high-power operational amplifiers. The direction of the motor is switched by BJT transistors. Lastly, the signal conditioning circuit consists on amplifiers and low-pass filters.

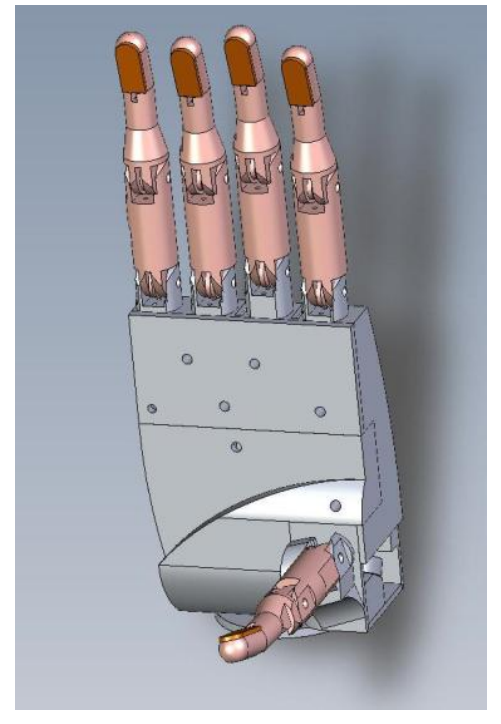

Figure 1. A CAD model of the prototype consisting on a five-fingered underactuated hand. It has anthropomorphic dimensions and weight similar to a real hand. Moreover, three active fingers are driven by a motor-gear system and spring system.

\subsection{Slip detection}

Since slip sensors are not commercially available for prosthetic applications, recent research had been focused on the development of their own sensors to incorporate the estimation of slippage information into prosthetic devices [7-9]. However, the final cost of the prosthesis using this kind of sensor can be affected enormously by the fabrication process. Consequently, we present a potential strategy here to detect slippage without using specialized sensors.
Using the information from the FSR, not only can we detect the level of normal force exerted by the prosthetic device, we can also estimate the onset of slippage between the fingers and the object. We proposed that slippage detection can be achieved by calculating the fluctuations of the reaction force by the object onto the active fingers. This approach was perceived when it was observed that a rapid change in the force was exhibited when slippage was produced. Once the hand has reached a stable grasping position, (when the fingers are not moving), the slip detection algorithm is activated. By calculating the absolute value of the derivative of the normal force at each sample point, we can quantify the rate of change of the force. The force signal was differentiated numerically at each sample point by the following five-point formula [10]:

$$
f^{\prime}\left(x_{0}\right)=\frac{1}{12}\left[f\left(x_{0}-2\right)-8 f\left(x_{0}-1\right)+8 f\left(x_{0}+1\right)-f\left(x_{0}+2\right)\right]
$$

Here, we are not concerned with how fast the object is sliding, but rather the onset of this activity. A threshold is empirically determined to determine the onset of the slippage.

Experiments consisting of grasping different objects and inducing slippage were performed. Pictures of different grasping patterns during slip detection experiments are showed in Figure 2. Data was collected at a sample frequency of $40 \mathrm{~Hz}$ using the NI DAQ cards (NI USB6009 National Instruments) and the algorithm was implemented in LabVIEW 8.2 (National Instruments).

\subsection{Force control}

In order to ensure stable grasping, a nonlinear control system was implemented to modulate the force exerted over the object during the Hold state. To illustrate the utility of the prosthetic device, different grasping configurations are tested. Some of these configurations are shown in Figure 2. As was mentioned above, the control technique employed was NMPC which its block diagram is illustrated in Figure 3 . Here $r$ is the force reference, $y$ is the system output (contact force), $\theta$ are the distal and the proximal angles, and $u$ is the motor current (control signal). The idea behind a general NMPC is to create a nonlinear model that estimates the dynamics of the system to predict future system output contact force $(\hat{y})$ over a defined time horizon. Afterwards, the controller uses this prediction to calculate the optimal input $(u)$, representing the applied motor current such that it follows the desired reference force $(r)$ over this 
horizon [11].

In our study, the model is implemented by a feedforward neural network (FFNN). Due to the ability of FFNN to approximate any nonlinear function (to an arbitrary accuracy level) [12], they are usually employed for nonlinear modelling. The structure of the FFNN was selected as autoregressive external input (NNARX) (see Figure 4). The network contains 15 inputs, 15 hidden neurons and 1 output. The activation function for the hidden layer is hyperbolic tangent and for the output layer is linear.

From the NNARX model the predictions are obtained by the FFNN output:

$$
\begin{aligned}
\hat{y}(t+i)=\mathbf{w}_{2} & {\left[\tanh \left(\mathbf{w}_{\mathbf{1}} \mathbf{p}+b_{1}\right)\right]+b_{2} } \\
\mathbf{p}= & {[y(t-3), \ldots, y(t-1), u(t-4), \ldots, u(t-1), \ldots} \\
& \left.\theta_{1}(t-4), \ldots, \theta_{1}(t-1), \theta_{2}(t-4), \ldots, \theta_{2}(t-1)\right]
\end{aligned}
$$

where $\mathbf{w}_{1}$ and $\mathbf{w}_{2}$ are the input-to-hidden and hidden-to-output weight matrixes respectively. The variables $b_{1}$ and $b_{2}$ are the bias terms and $\mathbf{p}$ is the input vector. By shifting $\mathbf{p}$ in time, the predictions in multiple time frames can be obtained.

Even though angle position is not one of the input parameters, it is strictly used as an observed quantify to assist in predicting the force for different grasping configuration.

Experiments were conducted using LabVIEW to obtain training and testing data to estimate the model of the finger system (this process is usually referred as system identification). The procedure consisted on closing the finger around different objects while applying an input signal to the motor. The input signal was designed (following [11]) as a chirp signal that is described as follows:

$$
\begin{aligned}
& u(t)=u_{o}+A \sin \left(\omega_{t} t\right) \\
& \omega_{t}=\omega_{\text {start }}+\frac{t}{N}\left(\omega_{\text {final }}-\omega_{\text {start }}\right)
\end{aligned}
$$

Different values of $u_{0}$ and $A$ along with different values of $\omega_{\text {start }}$ and $\omega_{\text {final }}$ were used in order to excite the whole range of the variables of the system [11]. The force and the two angles were measured for each active finger. These experiments were performed using various objects of different shapes and weights (i.e. bottles of different sizes, carton box and tennis ball, etc).

The signal amplitude of the training and testing data sets were scaled to zero-mean and unitary variance using the NNSYSID toolbox for Matlab (The
MathWorks) [13]. The synaptic weights of the neural network model was trained in an iteratively manner based on the Levenberg-Marquardt algorithm.

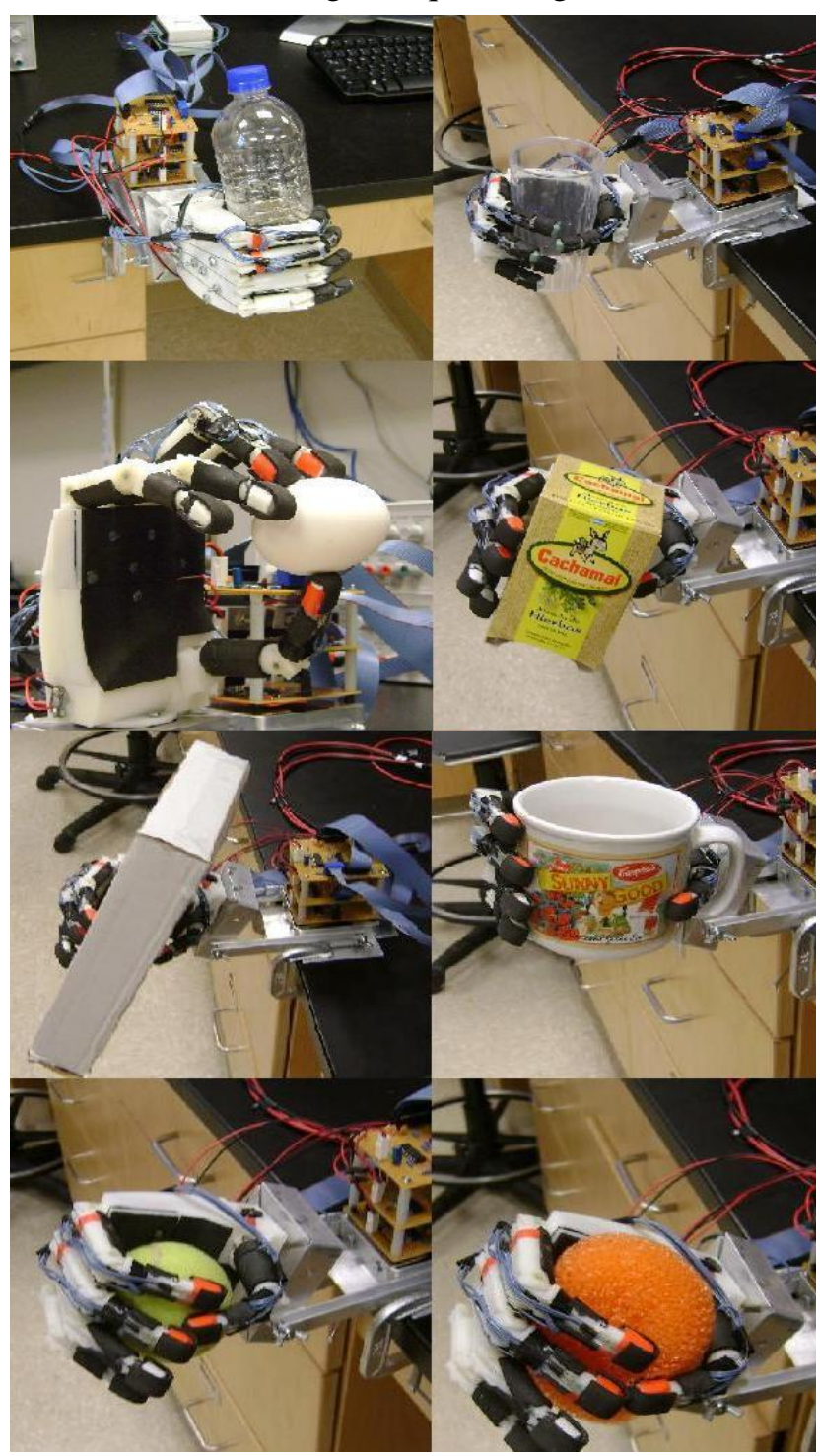

Figure 2. Different grasping patterns for the slippage experiments are illustrated.

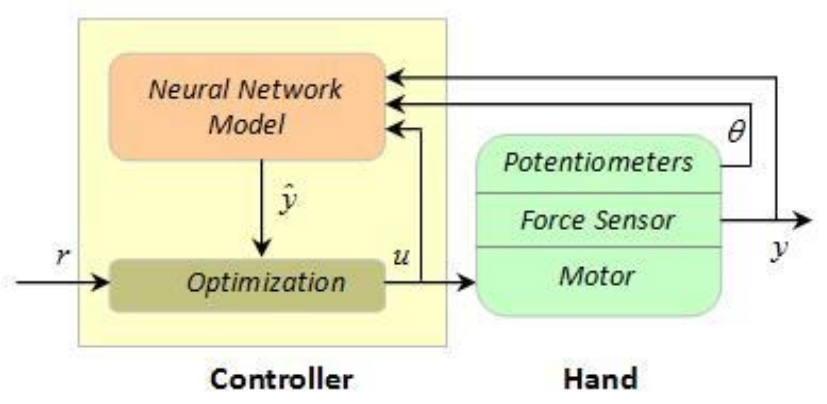

Figure 3. Block diagram of the NMPC strategy. The distal and proximal angles $\theta$ along with the actual contact force are feed to the neural network to predict future contact forces $\hat{y}$. Then, this information is used 
by the optimization block together with $r$ (the force reference), to compute $u$ (the motor current or control signal).

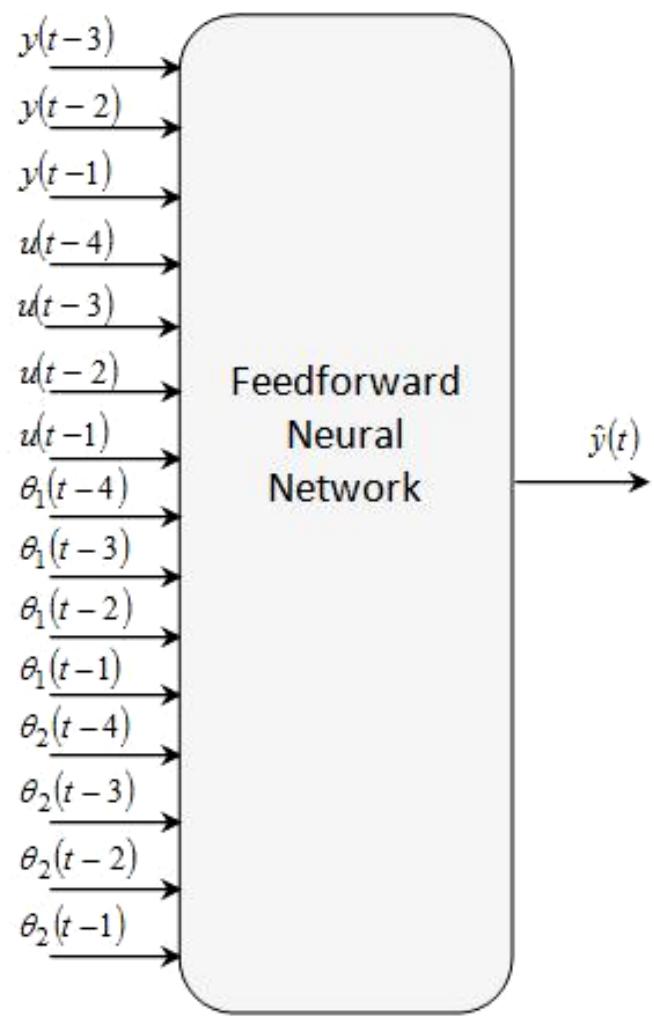

Figure 4. NNARX structure of the network. The applied current to the actuator, the joint angles and the force exerted on the object are used as the input to the feedforward neural network to estimate the future grasping force of the prosthetic device.

After training, the model was pruned using Optimal Brain Damage algorithm [11] using the NNSYSID toolbox. Afterwards, the testing error was checked. Sometimes if the sample frequency is too high compared to the dynamics of the system, a low test error would not necessarily translate to a good model [11]. Therefore, a second mode of analysis is required. The correlation tests were performed to validate the accuracy of the model. They are performed since the dynamics of the system has to be modeled such that the error is independent of the inputs and of the error itself.

For the optimization part of the controller, the objective function needs to be minimized with respect to the future control inputs:

$$
J(t, U(t))=\sum_{i=N_{1}}^{N_{2}}[r(t+i)-\hat{y}(t+i)]^{2}+\rho \sum_{i=1}^{N_{u}}[\Delta u(t+i-1)]^{2}
$$

$$
U(t)=\left[u(t) \cdots u\left(t+N_{u}-1\right)\right]^{T}
$$

where $N_{1}$ denotes the minimum prediction horizon, $N_{2}$ denotes the prediction horizon, $N_{u}$ the control horizon (after it the control input is considered constant) and $\rho$ is a weighting factor that restricts the changes in the control input [11].

The minimization of the objective function was implemented based on the methodology explained in $[11,14]$. It is performed by using gradient descendent technique where the future set of control inputs is determined by:

$U(t+1)=U(t)-\eta(t) \frac{\partial J}{\partial U(t)}$

where $\eta(t)$ is an adaptive learning rate of the following form:

$\eta(t)=\eta_{0} e^{[\alpha(r(t)-y(t))]}$

and $\alpha$ is a constant determined empirically. Then, if we express

$$
J(t, U(t))=E(t)^{T} E(t)+\rho \Delta U(t)^{T} \Delta U(t)
$$

we can expand the term $\frac{\partial J}{\partial U(t)}$ :

$$
\frac{\partial J}{\partial U(t)}=2 E(t) \frac{\partial \hat{Y}(t)}{\partial U(t)}+2 \rho \Delta U(t) \frac{\partial \Delta U(t)}{\partial U(t)}
$$

where:

$$
\begin{aligned}
& E(t)=\left[e(t+1), \ldots, e\left(t+N_{2}\right)\right]^{T} \\
& e(t+i)=r(t+i)-\hat{y}(t+i) ; \text { for } i=1, \ldots, N_{2} \\
& \hat{Y}(t)=\left[\hat{y}(t+1), \ldots, \hat{y}\left(t+N_{2}\right)\right]^{T} \\
& \Delta U(t)=\left[\Delta u(t), \ldots, \Delta u\left(t+N_{u}-1\right)\right]^{T}
\end{aligned}
$$

In addition, we have that

$$
\frac{\partial \Delta U(t)}{\partial U(t)}=\left[\begin{array}{ccccc}
1 & 0 & 0 & \cdots & 0 \\
-1 & 1 & 0 & \ddots & 0 \\
0 & \ddots & \ddots & \ddots & \vdots \\
\vdots & \ddots & -1 & 1 & 0 \\
0 & \cdots & 0 & -1 & 1
\end{array}\right]
$$

is a $N_{u} \times N_{u}$ matrix and 


$$
\frac{\partial \hat{Y}(t)}{\partial U(t)}=\left[\begin{array}{cccc}
\frac{\partial \hat{y}(t+1)}{\partial u(t)} & 0 & \cdots & 0 \\
\frac{\partial \hat{y}(t+2)}{\partial u(t)} & \frac{\partial \hat{y}(t+2)}{\partial u(t+1)} & \cdots & 0 \\
\vdots & \vdots & \ddots & \vdots \\
\frac{\partial \hat{y}\left(t+N_{2}\right)}{\partial u(t)} & \frac{\partial \hat{y}\left(t+N_{2}\right)}{\partial u(t+1)} & \cdots & \frac{\partial \hat{y}\left(t+N_{2}\right)}{\partial u\left(t+N_{u}-1\right)}
\end{array}\right]
$$

To calculate each element in Eq. (16), a recursive method is employed (for more details about this methodology please consult [14]). Furthermore, we designed the feedback control algorithm such that every time the system reaches a force within $\pm 1 \%$ of the reference force, the motor is turned off. This is implemented to avoid any undesirable oscillations and for reducing power consumption.

\section{Results and discussion}

\subsection{Slip detection}

Preliminary slippage-induced experiments for several objects with different shapes and weights were performed. Slippage was initially induced manually, where a person pulled the object in the direction perpendicular to the grasping force applied by the prototype. More quantitative experiments had since been developed and are now underway, in which the slippage is induced by a DC motor connected to a cable attached to the object. In this way the exact onset of the slippage can be known.

The result from one of the active fingers is illustrated in Figure 5. We observe a significant change in the normal force when slippage is induced. This is produced because of force redistribution at the tip of the finger. These fluctuations are represented in the force slope signal. The inclusion of a threshold is feasible due to a large difference in this force slope between the stable grasping state and the sliding state. It is also observed that only the first non-zero value in the slip signal is important given that it represents the onset of the sliding.

The top graph in Figure 5 shows the force signal recorded during the experiment while holding a small bottle. Firstly, stable grasp is reached, then slippage is induced after 5 seconds and stopped after 20 seconds such that stable grasp is produced again. The middle graph shows the absolute value of the derivative of the force signal. This graph also shows that the threshold was set arbitrarily to 0.01 , which was determined empirically. Finally, the bottom graph presents the slip signal resulting from the comparison between the slope of the force and the threshold. Similar results were obtained for others grasping configuration. We note in Figure 5 that a minimum level of force exerted over the object is necessary for the system to detect slippage. However, this level of force is small without causing deformation to the object.

\subsection{Force control}

Figure 6 illustrates the preliminary result of the feedback control for one of the active fingers. It shows that the prosthetic device was able to follow the different force reference levels while holding a small water bottle. The top graph shows the force reference signal and the actual force measured at the tip of the finger. The bottom graph shows the corresponding control signal (voltage supplied to the motor driver) during the same experiment. For this experiment the control parameters used were $N_{u}=3$, $N_{1}=1, N_{2}=3, \rho=0.2, \alpha=16$ and $\eta_{0}=5 \times 10^{-6}$. Note that the control signal was restricted between 4.8 and -2.7 volts.

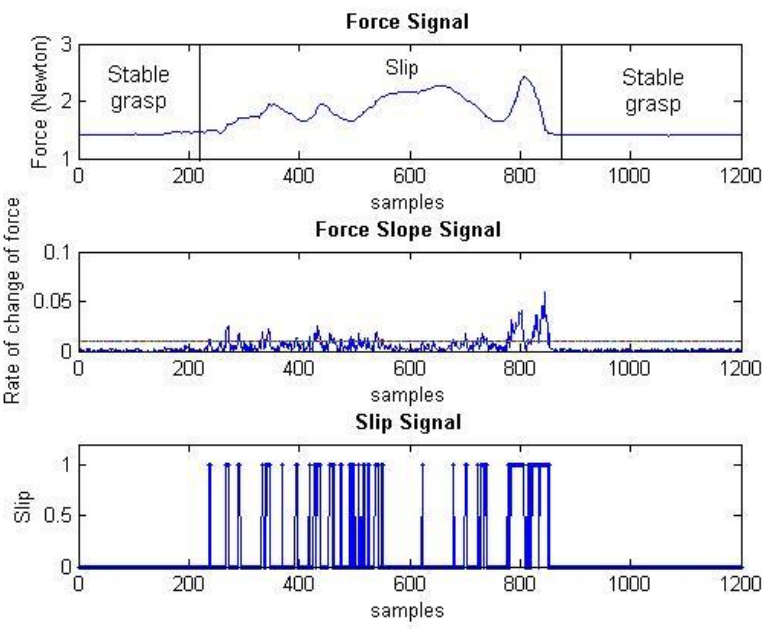

Figure 5. Top: sample of the force signal obtained from the index finger while holding a small bottle. Slippage is induced after 5 seconds and stopped after 20 seconds. Middle: absolute value of the derivative of the force and setting of the threshold. Bottom: Slip signal (1: slip; 0: nonslip).

In general, the system follows the reference signal with acceptable accuracy (around 1\% of the reference value). The system occasionally overshoots the reference value during the control phase; however these overshoots are not sufficiently large to cause any damage. In addition, time delays can be observed for some reference values, which can affect the reaction time of the hand. This effect is produced mainly due to the dead band of the DC motor and also to the PWM operating range and it is depended on the learning rate 
of the algorithm. In addition, it is necessary to maintain this delay as small as possible in order for the system to grasp the object securely.
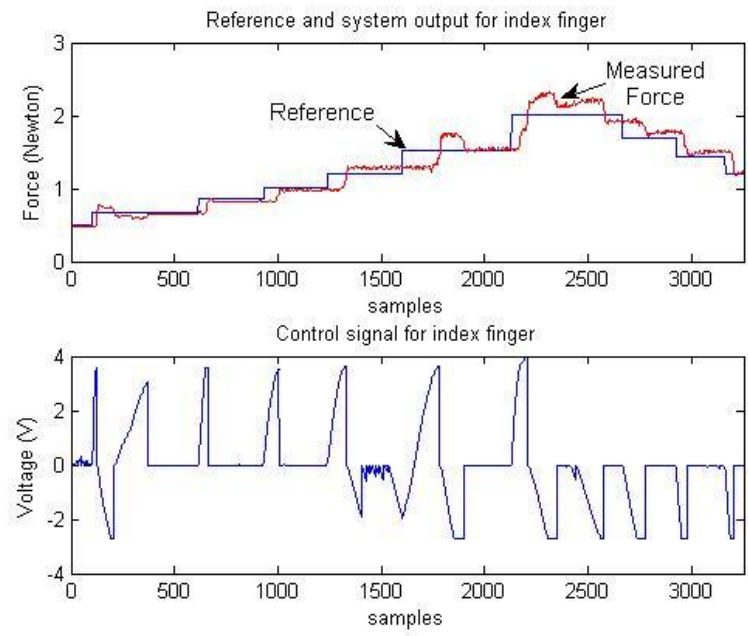

Figure 6. Top: reference and output signal during experiment with a small bottle. Bottom: control signal (voltage supplied to the motor).

\section{Conclusion}

In this paper a low-cost mechatronic system for a prosthetic hand was presented. It intends to bridge a translational gap between the technology and commercialization and utility to bring a possible solution for reducing the cost of prosthesis. Moreover, a prototype along with a control strategy was described with promising results showing the possibility of slippage detection and the force controller for each finger. Some issues must be addressed in order to improve the performance of the system. At this moment, the finger is been controlled in a predictive fashion, based on a static model. However, it is important to make the model to approach asymptotically to the real system in order to better represent the system dynamics [14]. This added precision will result in better performance of the control system. Consequently, the incorporation of an adaptive algorithm such that the model can be dynamically modified in real time is being considered as a next step of the controller design. Currently, the control system uses the information from the gradient of the objective function to compute the control signal. It is well known that this method is slow compared with any Hessian-based algorithms for nonlinear optimization. Therefore, the implementation of an optimization algorithm based on the second derivative of the objective function is now underway.

Am. J. Biomed. Sci. 2009, 1(4), 295-302; doi: 10.5099/aj090400295
Finally, at present the force at each finger is controlled independently. Future work consists of integrating the control system for each finger in one general control strategy, incorporating at the same time the slippage information.

\section{References}

1. Carrozza, M.C.; Cappiello, G.; Beccai, L.; Zaccone, F.; Micera, S.; Dario, P.; Design methods for innovative hand prostheses, Conf Proc IEEE Eng Med Biol Soc., 2004, 6, 4335-4348.

2. Cipriani, C.; Zaccone, F.; Stellin, G.; Beccai, L.; Cappiello, G.; Carrozza, M.C.; Dario, P.; Closedloop controller for a bio-inspired multi-fingered underactuated prosthesis. Proc. Of IEEE International Conference on Robotics and Automation. 2006.

3. Light, C.M.; Chappell, P.H.; Hudgins, B.; Engelhart, K.; Intelligent multifunction myoelectric control of hand prostheses. Journal of Medical Engineering and Technology, 2002. 26(4), 139-146.

4. Rodriguez-Cheu, L.E.; Gonzalez, D.; Rodriguez, M.; Result of a perceptual feedback of the grasping forces to prosthetic hand users. Biomedical Robotics and Biomechatronics, 2008, 901-906.

5. Birglen, L.; Gosselin, C.M.; Fuzzy enhanced control of an underactuated finger using tactile and position sensors. Proc. Of IEEE International Conference on Robotics and Automation. 2005.

6. Engeberg, E.D.; Meek, S.; Improved Grasp Force Sensitivity for Prosthetic Hands Through ForceDerivative Feedback. IEEE Transactions on Biomedical Engineering, 2008. 55(2), 817-821.

7. Cotton, D.P.J.; Cranny, A.; White, N.M.; Chappell, P.H.; Beeby, S.P.; A novel thick-film piezoelectric slip sensor for a prosthetic hand. IEEE Sensors Journal, 2007. 7(5), 752-761.

8. Wettels, N.; Popovic, D.; Santos, V.J.; Johannsson, R.S.; Loeb, G.E.; Biomimetic tactile sensor for control of grip. IEEE International Conference on Rehabilitation Robotics, 2007, 923-932.

9. Kyberd, P.J.; Evans, M.; te Winkel, S.; An intelligent anthropomorphic hand with automatic grasp. Robotica, 1998. 16(5), 531-536.

10. Burden, R.L.a.F., J. D., Numerical Analysis. 8 ed., Brooks Cole, 2004.

11. Norgaard, M.; Ravn, O.; Poulsen, N.K.; Hansen, L.K.; Neural Networks for Modelling and Control of Dynamic Systems: A Practitioner's Handbook. Advanced Textbooks in Control and Signal Processing, Springer, 2000.

(c) 2009 by NWPII. All rights reserved. 
12. Hornik, K.; Stinchcombe, M.; White, H.; Multilayer feedforward networks are universal approximators. Neural Networks, 1989. 2(5), 359366.

13. Norgaard, M.; Ravn, O.; Poulsen, N.K.; NNSYSID and NNCTRL tools for system identification and control with neural networks. Computing \& Control Engineering Journal, 2001. 12(1): 29-36.
14. Noriega, J.R.; Wang, H.; A direct adaptive neuralnetwork control for unknown nonlinear systems and Its application. IEEE Transactions on Neural Networks, 1998. 9(1): 27-34. 